Draft Version November 2, 2018

Preprint typeset using LATEX style emulateapj v. 02/07/07

\title{
MODELING FLOWS AROUND MERGING BLACK HOLE BINARIES
}

\author{
James R. van Meter ${ }^{1}$, John H. Wise ${ }^{2}$, M. Coleman Miller ${ }^{3}$, Christopher S. Reynolds ${ }^{3}$, Joan Centrella ${ }^{1}$, John \\ G. Baker ${ }^{1}$, William D. Boggs ${ }^{1},{ }^{4}$, Bernard J. Kelly ${ }^{1}$, and Sean T. McWilliams ${ }^{1}$ \\ Draft version November 2, 2018
}

\begin{abstract}
Coalescing massive black hole binaries are produced by the mergers of galaxies. The final stages of the black hole coalescence produce strong gravitational radiation that can be detected by the spaceborne LISA. In cases where the black hole merger takes place in the presence of gas and magnetic fields, various types of electromagnetic signals may also be produced. Modeling such electromagnetic counterparts of the final merger requires evolving the behavior of both gas and fields in the strong-field regions around the black holes. We have taken a step towards solving this problem by mapping the flow of pressureless matter in the dynamic, 3-D general relativistic spacetime around the merging black holes. We find qualitative differences in collision and outflow speeds, including a signature of the merger when the net angular momentum of the matter is low, between the results from single and binary black holes, and between nonrotating and rotating holes in binaries. If future magnetohydrodynamic results confirm these differences, it may allow assessment of the properties of the binaries as well as yielding an identifiable electromagnetic counterpart to the attendant gravitational wave signal.

Subject headings: black hole physics — galaxies: nuclei — gravitational waves
\end{abstract}

\section{INTRODUCTION}

Electromagnetic signatures of the coalescence of two supermassive black holes would, in concert with the detection of gravitational waves from the event, allow a high-precision determination of both the redshift and the luminosity distance to the merger. This would provide a precise probe of cosmology, limited mainly by uncertainties in the magnification, and hence the luminosity distance, due to weak gravitational lensing (Hughes \& Holz 2003). As a result, there has been substantial recent interest in mechanisms that could produce electromagnetic emission from a surrounding accretion disk Armitage \& Natarajan 2002; Milosavliević \& Phinnev 2005; Dotti et al. 2006; Kocsis et al. 2006; Phinnev 2007; Bode \& Phinnev 2007; Shields \& Bonning 2008; Lippai et al. 2008; Schnittman \& Krolik 2008; Kocsis \& Loeb 2008; Kocsis et al. 2008; Haiman et al. 2009; O'Neill et al. 2009; Haiman et al. 2009; Phinnev 2009; Chang et al. 2009; Megevand et al. 2009). However, there have been relatively few investigations of the electromagnetic signatures that could emerge from the dynamic spacetime near the binary during its last few orbits (for a recent exception, see Palenzuela et al. 2009).

There are reasons to believe that if significant gas exists around the binary in its last few orbits, then intense but short-lived electromagnetic signals will be produced. For example, since the binary orbital speed can be half the speed of light or more, even in the Newtonian case the slingshot mechanism could be particularly effective

\footnotetext{
Electronic address: james.r.vanmeter@nasa.gov

${ }^{1}$ Gravitational Astrophysics Laboratory, NASA Goddard Space Flight Center, Greenbelt, MD 21114

${ }_{2}^{2}$ Laboratory for Observational Cosmology, NASA Goddard Space Flight Center, Greenbelt, MD 21114

${ }^{3}$ University of Maryland, Department of Astronomy, College Park, MD 20742

${ }^{4}$ University of Maryland, Department of Physics College Park, MD 20742
}

in ejecting matter or producing collisions that have a high Lorentz factor. In addition, it has been proposed that magnetic fields in the vicinity of the orbit could be wound up and amplified to such a degree that they would produce a strong Poynting outflow (R. Blandford, personal communication). Exploration of such effects would require simulations that involve magnetohydrodynamics (MHD) in a dynamical spacetime.

Here we take a step towards such simulations by tracing the paths of pressureless test particles (i.e., particles of non-zero rest mass in geodesic motion) in the dynamical spacetime of coalescing black holes. Our goal is to determine whether the outflows and collisions are sufficiently high-speed, compared with the equivalents around a single black hole, that unique signatures of the binary motion seem plausible. This will pave the way for the extensive development required to do a full analysis of MHD in a dynamical spacetime. In $\S 2$ we discuss our simulation setup, which uses a code that has been used to model successfully the coalescence of black holes of different masses, spin magnitudes, and orientations. We choose units in which $c=1$ and $G=1$; with this, the total mass $M$ is related to time and distance by $M \sim 5 \times 10^{-6}\left(M / M_{\odot}\right) \mathrm{s} \sim 1.5\left(M / M_{\odot}\right) \mathrm{km}$. In $\S 3$ we present and discuss our results, in which we examine first the coalescence of equal-mass nonspinning black holes and then show that equal-mass black holes with aligned spins produce significantly more extreme effects.

\section{SIMULATION SETUP}

In the following simulations the spacetime was computed by solving Einstein's field equations using finite differencing methods of numerical relativity (Imbiriba et al. 2004; Baker et al. 2008). On the initial slice, the black holes are represented by "punctures" (Brandt \& Brügmann 1997) and the constraint equations are solved using a multigrid solver (Brown \& Lowe 2005). The black holes are then evolved forward in time by integrating the time-dependent Einstein equations 
given by the BSSN formulation (Shibata \& Nakamura 1995; Baumgarte \& Shapiro 1998). We use coordinate conditions that allow the black holes to move freely across the grid (Baker et al. 2006; Campanelli et al. 2006; van Meter et al. 2006). Meanwhile the test matter is modeled by non-interacting point-particles. To evolve the test matter, we integrate the geodesic equation for each particle in this spacetime. The metric at the location of each particle is obtained by interpolation from the computational grid.

The geodesic code was tested for a variety of trajectories around a single black hole, both spinning and nonspinning. For coordinate conditions in which the conversion to Boyer-Lindquist coordinates was known, the particle worldline was compared with independent calculations and found to be at least 4th-order convergent in the grid-spacing, as were the constants of motion, energy $(E)$, angular momentum $(L)$, and Carter's constant $(Q)$. For the coordinate conditions closer to those of our typical binary black hole runs, the constant of motion $L$, readily calculable in any axisymmetric coordinate system, was still found to be at least 4th-order convergent.

Initial data for the spacetime background used here was chosen to represent two black holes, each of mass $m=M / 2$, in nearly circular inspirals. In our simulations the black holes were either spinless, or were each given a spin of $a / m=0.8$, aligned with the orbital angular momentum. The initial linear momenta of the black holes were given by the post-Newtonian approximation, and the initial separation of the black holes was chosen so as to give at least five orbits before merger. As a control case, we have also run equivalent simulations with a single isolated nonrotating black hole of mass $M$.

In these spacetimes, approximately 75,000 geodesic particles were initially distributed uniformly throughout a solid annulus of inner radius $8 M$, outer radius $25 M$, and vertical full thickness $10 M$. We excluded particles within the inner radius to avoid transient signatures from particles initially near the horizons. We have chosen such a geometrically thick disk because these are the disks that potentially have high enough inward radial speeds to keep up with the shrinking binary, as opposed to being stranded at large radii as the binary coalesces (see Milosavliević \& Phinnev 2005).

We explored two initial velocity configurations for the particles. In the first configuration ("orbital"), the initial velocities are randomly distributed around a tangential velocity $V_{c}$ that would give a circular orbit in a Schwarzschild spacetime of mass $M$, resulting in a scale height of $5 \mathrm{M}$. In the second configuration ("isotropic"), we consider an extreme case where the particles only have random velocities, with each component sampled from a Gaussian distribution of standard deviation $V_{c} / \sqrt{3}$.

We output the positions and proper 4-velocities of the particles every $\sim 1 M$ and $\sim 0.5 M$ in the nonrotating and rotating cases, respectively. Particles within a horizon are discarded. We use the relative velocities between two "colliding" particles as an energy estimate of the material within the disk. We define a particle collision as two particles traveling within $r_{\mathrm{c}}=0.1 M$ of each other, assuming that they travel on straight lines between successive output times. We have found that the collision energies are not sensitive to $r_{\mathrm{c}}$ in the range of $0.01 M$ to

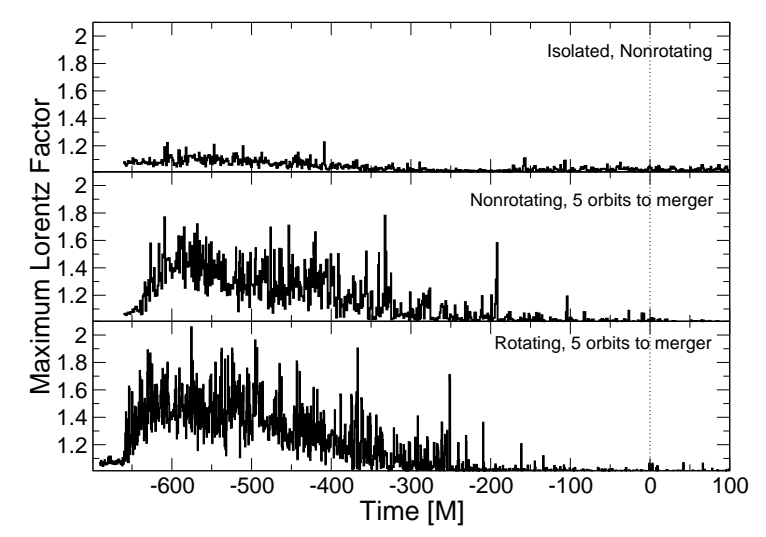

Fig. 1.- Maximum Lorentz factors of collisions between particles as function of time before merger for initial velocities that include an orbital velocity plus a random isotropic component. The three panels are for the following cases: (top) isolated nonrotating black hole, (middle) two spinless black holes, merging in approximately five orbits, (bottom) two black holes each with spin parameters of $a / m=0.8$, merging in approximately six orbits. In all cases both black holes have mass $m$. The data have been aligned with respect to the time axis such that the common apparent horizon is first detected at $t=0 . M$ is the total system mass.

$1 M$. For every particle, we search the nearest 8 neighbors for any particle that has a closest approach less than $r_{\mathrm{c}}$, which we mark as a collision.

With this list of collisions, we can now obtain the Lorentz factor of each colliding particle, in locally Minkowskian coordinates, as follows. Given two colliding particles $A$ and $B$, first we compute the scalar product of the proper 4 -velocity $u_{A}^{\mu}$ of particle $A$ with the proper 4 -velocity $u_{B}^{\mu}$ of particle $B$. Note that regardless of the original coordinates in which the product is computed, it exactly equals the negative of the Lorentz factor of particle $A$ in the rest-frame of particle $B$ (as it would be calculated in locally Minkowskian coordinates). That is,

$$
u_{A}^{\mu} u_{B}^{\nu} g_{\mu \nu}=u_{A}^{\mu^{\prime}} u_{B}^{\nu^{\prime}} g_{\mu \nu}^{\prime}=-u_{A}^{0^{\prime}}
$$

because $u_{B}^{\mu^{\prime}}=\delta_{0}^{\mu}$ and $g_{\mu \nu}^{\prime}=\eta_{\mu \nu}$, where the primes refer to the inertial frame of particle $B$. We then transform to the center-of-momentum frame by solving for the Lorentz factor $\gamma_{\text {com }}$ characterizing the Lorentz transformation matrix that transforms $u_{A}^{\mu^{\prime}}$ and $u_{B}^{\mu^{\prime}}$ to $u_{A(\text { com })}^{\mu}$ and $u_{B(\text { com })}^{\mu}$ such that

$$
u_{A(\mathrm{com})}^{0}=u_{B(\mathrm{com})}^{0}=\gamma_{\mathrm{com}}, u_{A(\mathrm{com})}^{i}=-u_{B(\mathrm{com})}^{i} .
$$

We finally obtain:

$$
\gamma_{c o m}=\sqrt{\frac{1}{2}\left(1-u_{A}^{\mu} u_{B}^{\nu} g_{\mu \nu}\right)} .
$$

\section{RESULTS AND DISCUSSION}

Our main results are displayed in Figures 1 through 4. In Figures 1 and 2, with initially orbital and isotropic particle velocities respectively, we show the maximum Lorentz factor of a collision between any two particles as a function of time, for several different setups including one with an isolated Schwarzschild black hole for comparison. Because collisions do not affect particle trajectories in our calculations (i.e., the geodesic paths are unaltered), we expect these to be upper bounds on the true Lorentz factors of collisions given that in the MHD 


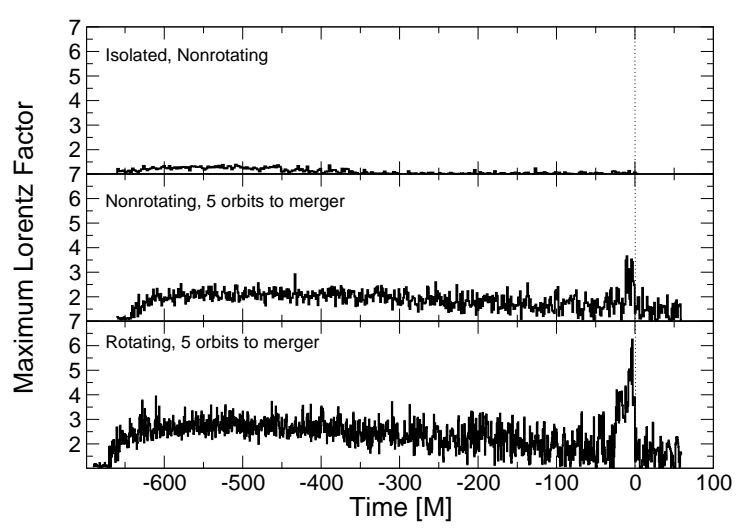

FIG. 2.- Same as Figure1 1but for particles whose initial velocity is purely random and isotropic, with no net orbital component. From this figure we note that (a) in both binary cases there is a clear maximum in Lorentz factor just prior to merger, whereas (as expected) the isolated case has a steady Lorentz factor, and (b) in the spinning case, the collisions throughout and near merger are more violent than in the nonrotating runs.

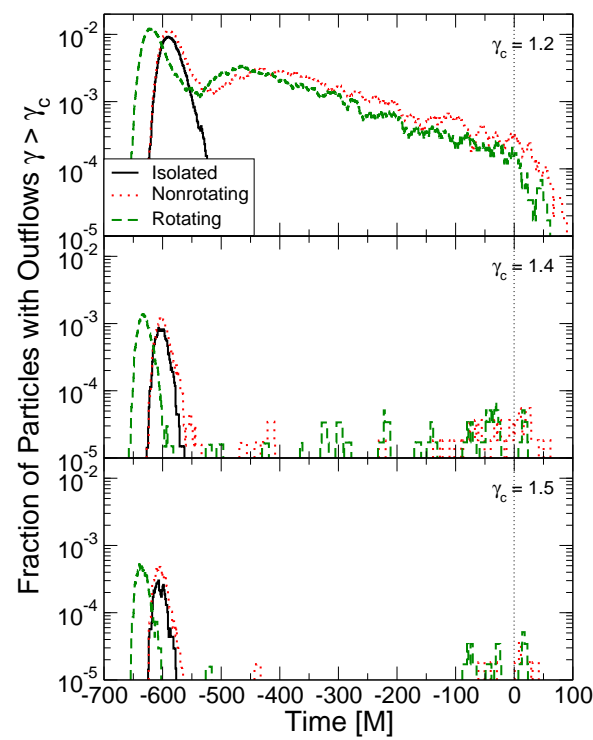

FIG. 3. - The fraction of particles with positive radial velocities in a shell with surfaces at $25 M$ and $30 M$ with a corresponding Lorentz factor greater than (top to bottom) 1.2, 1.4, 1.5, comparing the cases with an isolated spinless black hole (solid black), a binary with spinless equal-mass black holes (dotted red), and a binary with equal-mass black holes with spins of $a / m=0.8$ (dashed green). On the scale plotted, one particle gives a fractional value of $1 / 75,000$ or $1.3 \times 10^{-5}$.

situation realized by nature there will be dissipation of relative speeds through collisions.

In Figures 1 and 2, the top panel shows the control case, in which the particles orbit around a solitary black hole. The other two panels involve nonspinning (middle panel) and spinning (bottom panel) binaries. The merger in these cases, defined by the detection of a common apparent horizon, takes place at $t=0$.

Our control case shows as expected that after a short initialization phase the maximum Lorentz factor is roughly constant. Here the highest-speed collisions are between particles that have both fallen close to the horizon and thus have acquired speeds considerably in excess of their initial orbital speeds. Given that the particles have a "thermal" velocity distribution in which the random speed is comparable to the orbital speed, a rea-

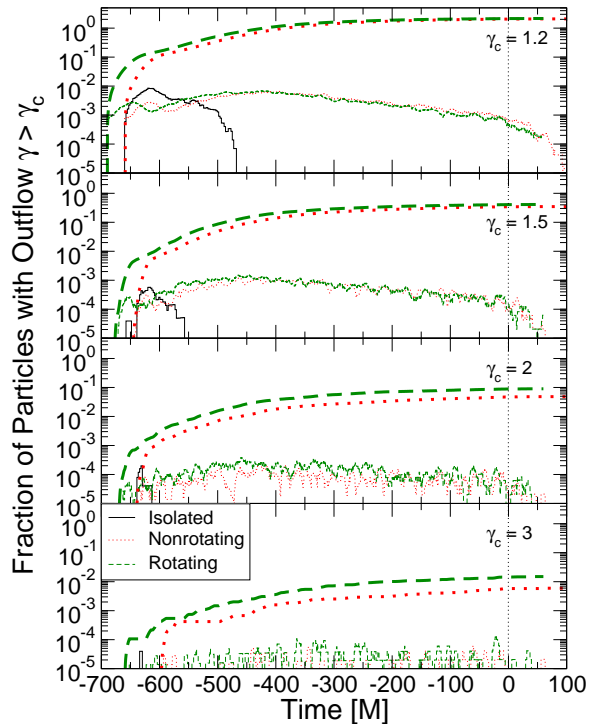

FIG. 4. - Same as Figure 3 but for the case of particles having only initial random velocities and Lorentz factors greater than (top to bottom) $1.2,1.5,2,3$. Also shown are the cumulative sums of these fractions.

sonable fraction of particles is captured by the black hole.

In the binary runs the varying accelerations caused by the binary motion are expected to alter significantly the energy and angular momentum of particles within $\sim 2$ binary orbital radii, just as in the Newtonian slingshot analogue. More rapid orbits therefore are expected to lead to higher-speed collisions and (as is evident in Figure 4) faster outflows, with the effect maximized shortly before merger because this is when the orbital speeds are greatest.

Examining Figure 2 we find that these expectations are largely met for the isotropic particle velocities. There is indeed a strong peak in the maximum collision Lorentz factor just before merger. This peak is highest when the holes are rotating, as is reasonable because for the aligned spin configuration the holes can get closer and thus orbit faster before they plunge together. The Lorentz peaks are in all cases considerably higher than in the isolated hole runs, which might lead us to cautious optimism that this phase might be observably different for a binary merger than for an isolated hole. However, in the case of particles with initial average velocities that are approximately orbital, the central region of the disk remains evacuated near the time of merger, as the particles have found quasi-stable orbits further out. In this case there is no discernible merger signature, as seen in Figure1. We conjecture that the inclusion of viscosity would push orbiting matter closer towards the binary, resulting in energetics between the above two extremes, and recovering a merger signature. If this is confirmed by future detailed MHD calculations, it may provide another way to identify an electromagnetic counterpart to the gravitational waves from a binary supermassive black hole merger.

Other features of these figures are, we believe, artifacts of the limitations of our simulation. For example, even with isotropic particle velocities, in both the spinning and the nonspinning binary runs we see an overall decrease in the maximum Lorentz factor of collisions during inspiral. We suspect that this is because our setup 
includes no angular momentum transport between particles, hence as the simulation progresses we run out of particles that would naturally plunge towards the holes and produce very high speed collisions.

In Figure 3 and 4 we explore the outflow Lorentz factor, measured in a shell of thickness $5 M$ with its inner surface equal to initial disk outer radius of $25 \mathrm{M}$. Particularly in the case of isotropic particle velocities, because we have a significant random speed in our thick disks, the isolated hole has some fraction of particles that move rapidly outward, but we see that the fraction of such particles is (as expected) very small compared to those of the binary runs where particles are flung out via a slingshot effect.

The main meaningful difference, in Figure 4, is between the spinning and nonspinning binary simulations. At a given time before merger, due to spin-orbit coupling in the spinning case, the holes are orbiting faster than in the nonspinning case. As a result, the slingshot effect is stronger at all times from merger, and near merger itself the speeds are higher yet. It is also possible that framedragging due to spin plays a more direct role, by increasing the ejection speeds in a manner akin to an eggbeater. At the highest speeds seen, there is no parallel for the isolated black hole run. Depending greatly on the nature of the interactions of the outflow (e.g., the development of shocks or the initiation of Fermi acceleration), it is possible that high energy radiation such as gamma-rays may be produced in ways that make it possible to discriminate between single and binary black holes, or even possibly between slowly-rotating and rapidly-rotating black holes.

In summary, our exploratory calculations represent a step towards realistic simulations of the accretion flow near binary supermassive black holes close to merger. Under certain conditions our results show differences between single and binary black holes, and between nonrotating and rapidly rotating binary black holes, which is encouraging for future observations. As such, they motivate more realistic hydrodynamic and magnetohydrodynamic calculations, which will be our next steps. If some of the qualitative differences found in our test particle calculations (with isotropically distributed velocities) are maintained in future work with fluids, this will imply prompt electromagnetic signatures that can be correlated with gravitational radiation signals detected by the space-based Laser Interferometer Space Antenna (Baker et al. 2007; Lang \& Hughes 2009), allowing precise cosmological probes and constraints on dark energy (Arun et al. 2009) and even testing fundamental principles such as the relative speed of propagation of photons and gravitational waves.

We performed these calculations on Discover at NASA / GSFC and Pleiades at NASA / AMES. CSR and MCM acknowledge partial support from the National Science Foundation under grant AST 06-07428. MCM was also supported in part by NASA ATP grant NNX08AH29G. The work at Goddard supported in part by NASA grant 06-BEFS06-19. JHW, STM and BJK were supported by appointments to the NASA Postdoctoral Program at the Goddard Space Flight Center, administered by Oak Ridge Associated Universities through a contract with NASA. 


\section{REFERENCES}

Armitage, P. J., \& Natarajan, P. 2002, ApJ, 567, L9

Arun, K. G., et al. 2009, Class. Quant. Grav., 26, 094021

Baker, J. G., Boggs, W. D., Centrella, J. M., Kelly, B. J., McWilliams, S. T., \& van Meter, J. R. 2008, Phys. Rev. D, 78, 044046

Baker, J. G., Centrella, J. M., Choi, D.-I., Koppitz, M., \& van Meter, J. R. 2006. Phys. Rev. Lett., 96, 111102

Baker, J. G., et al. 2007, LISA: Probing http://www.lisa-science.org/resources/talks-articles/ science/lisa_science_case.pdf

Baumgarte, T. W., \& Shapiro, S. L. 1998, Phys. Rev. D, 59, 024007

Bode, N., \& Phinney, S. 2007, APS Meeting Abstracts, 1010

Brandt, S. R., \& Brügmann, B. 1997, Phys. Rev. Lett., 78, 3606

Brown, J. D., \& Lowe, L. L. 2005, J. Chem. Phys., 209, 582

Campanelli, M., Lousto, C. O., Marronetti, P., \& Zlochower, Y. 2006, Phys. Rev. Lett., 96, 111101

Chang, P., Strubbe, L. E., Menou, K., \& Quataert, E. 2009, arXiv:0906.0825

Dotti, M., Salvaterra, R., Sesana, A., Colpi, M., \& Haardt, F. 2006, MNRAS, 372,869

Haiman, Z., Kocsis, B., \& Menou, K. 2009, ApJ, 700, 1952

Haiman, Z., Kocsis, B., Menou, K., Lippai, Z., \& Frei, Z. 2009 Classical and Quantum Gravity, 26, 094032

Hughes, S. A., \& Holz, D. E. 2003, Classical and Quantum Gravity, 20,65
Imbiriba, B., Baker, J. G., Choi, D.-I., Centrella, J. M., Fiske, D. R., Brown, J. D., van Meter, J. R., \& Olson, K. 2004 Phys. Rev. D, 70, 124025

Kocsis, B., Frei, Z., Haiman, Z., \& Menou, K. 2006, ApJ, 637, 27 Kocsis, B., Haiman, Z., \& Menou, K. 2008, ApJ, 684, 870

Kocsis, B., \& Loeb, A. 2008, Physical Review Letters, 101, 041101 Lang, R. N., \& Hughes, S. A. 2009, Classical and Quantum Gravity, 26,094035

Lippai, Z., Frei, Z., \& Haiman, Z. 2008, ApJ, 676, L5

Megevand, M., et al. 2009, Phys. Rev. D, 80, 024012

Milosavljević, M., \& Phinney, E. S. 2005, ApJ, 622, L93

O'Neill, S. M., Miller, M. C., Bogdanovic, T., Reynolds, C. S., \& Schnittman, J. D. 2009, ApJ, 700, 859

Palenzuela, C., Anderson, M., Lehner, L., Liebling, S. L., \& Neilsen, D. 2009, arXiv:0905.1121

Phinney, E. S. 2007, APS Meeting Abstracts, 7002

-. 2009, Finding and Using Electromagnetic Counterparts of Gravitational Wave Sources, Astronomy 2010 decadal whitepaper no. 235

Schnittman, J. D., \& Krolik, J. H. 2008, ApJ, 684, 835

Shibata, M., \& Nakamura, T. 1995, Phys. Rev. D, 52, 5428

Shields, G. A., \& Bonning, E. W. 2008, ApJ, 682, 758

van Meter, J. R., Baker, J. G., Koppitz, M., \& Choi, D.-I. 2006, Phys. Rev. D, 73, 124011 